\title{
COMPATIBLE SYSTEMS OF MOD $p$ GALOIS REPRESENTATIONS AND HECKE CHARACTERS
}

\author{
Chandrashekhar Khare
}

\section{Introduction}

Compatible systems of $n$-dimensional, $\bmod p$ representations of absolute Galois groups of number fields were considered by Serre in his study of openness of images of adelic Galois representations arising from elliptic curves in [S2]. In $[\mathrm{K}]$ the author considered abstract compatible systems of $n$-dimensional, $\bmod p$ representations of the absolute Galois group of $\mathbf{Q}$ and determined them in the one-dimensional case.

1.1. Definition and main theorem. We begin with the definition of compatible systems of $n$-dimensional, $\bmod p$ representations of the absolute Galois group of a number field $K$.

Definition 1. Let $K$ and $L$ be number fields and $S, T$ finite sets of places of $K$ and $L$ respectively. An $L$-rational (resp., L-integral) strictly compatible system $\left\{\rho_{\wp}\right\}$ of $n$-dimensional mod $\wp$ representations of $G_{K}:=\operatorname{Gal}(\bar{K} / K)$ with defect set $T$ and ramification set $S$, consists of giving for each finite place $\wp$ of $L$ not in $T$ a continuous, semisimple representation

$$
\rho_{\wp}: G_{K} \rightarrow G L_{n}\left(\mathbf{F}_{\wp}\right),
$$

for $\mathbf{F}_{\wp}$ the residue field of $\mathcal{O}_{L}$ at $\wp$ of characteristic $p$, that is

- unramified at the places outside $S \cup\{$ places of $K$ above $p$ \}

- there is a monic polynomial $f_{r}(X) \in L[X]$ (resp., $f_{r}(X) \in \mathcal{O}_{L}[X]$ ) such that for each place $r$ of $K$ not in $S$ and for all places $\wp$ of $L$ not in $T$, coprime to the residue characteristic of $r$, and such that $f_{r}(X)$ has coefficients that are integral at $\wp$, the characteristic polynomial of $\rho_{\wp}\left(\right.$ Frob $\left._{r}\right)$ is the reduction of $f_{r}(X) \bmod \wp$, where Frob $r$ is the conjugacy class of the Frobenius at $r$ in the Galois group of the extension of $K$ that is the fixed field of the kernel of $\rho_{\wp}$.

In the course of the paper we will often suppress the sets $S, T$ from the notation. It might be prudent to impose the condition of integrality of the roots of $f_{r}(X)$ at primes coprime to the residue characteristic of primes in $T$ and $r$

Received October 30, 2001.

Revised version received September 24, 2002. 
in Definition 1. Then the $\rho_{\wp}$ 's are determined a priori by the compatible system data $\left\{f_{r}(X)\right\}$. But as we do not need this in the main result of the paper, Theorem 1 below, we stick to our less stringent requirements.

One would like to prove that a strictly compatible system arises motivically, i.e., "from the $\bmod p$ étale cohomology of a variety $X_{/ K}$ as $p$ varies". In the case of one-dimensional strictly compatible systems this is interpreted as saying that it arises from a Hecke character. It is only in the one-dimensional case thanks to class field theory that at the moment one has a realistic chance of describing compatible sytems in any degree of generality. We do this below and prove in Section 4.2 the following theorem that is the main result of the paper (for any unexplained terms see Section 4.1, in particular Definition 4).

Theorem 1. An L-rational strictly compatible system $\left\{\rho_{\wp}\right\}$ of one-dimensional mod $\wp$ representations of $\operatorname{Gal}(\bar{K} / K)$ arises from a Hecke character.

Corollary 1. An L-rational strictly compatible system of one-dimensional $\wp-$ adic representations $\left\{\rho_{\wp, \infty}\right\}$ as in I-11 of [S] arises from a Hecke character.

Proof of Corollary 1. Observe that, given a strictly compatible system of $\wp$-adic representations as in I-11 of $[\mathrm{S}]$, if we reduce it $\bmod \wp$ and semisimplify, we get a rational strictly compatible system in the sense of Definition 1 such that $f_{r}(X)$ is integral away from the primes that have the same residue characteristic as $r$. The observation follows from the fact that continuous $\wp$-adic representations of the group $G_{K}$ have integral models as $G_{K}$ is compact. Using the observation we see that the resulting strictly compatible system of mod $\wp$ representations determines the system of $\wp$-adic representations. From this observation and Theorem 1, the corollary follows.

The corollary is Proposition 1.4 in [Sch]. It is deduced there as a consequence of the much stronger result proved in $[\mathrm{H}]$ that even a single, algebraic, one-dimensional $\wp$-adic representation arises from a Hecke character. The proof in $[\mathrm{H}]$ requires deep results of Waldschmidt in transcendental number theory. The corollary in a weaker form, assuming a supplementary purity hypothesis, is also an old theorem of Taniyama (see Theorem 1 of [Tani]) that he proved using methods quite different from the present paper. But both in [Tani] and in the present work the compatibility hypotheses are used to describe 1-dimensional compatible systems using purely algebraic methods, and Theorem 1 can be addressed using only such methods.

We point out a corollary that is immediate from the theorem but that sets us up to formulate two conjectures in the higher dimensional case.

Corollary 2. An L-rational strictly compatible system $\left\{\rho_{\wp}\right\}$ of one-dimensional mod $\wp$ representations of $\operatorname{Gal}(\bar{K} / K)$ lifts to a strictly compatible system of onedimensional $\wp$-adic representations as in I-11 of [S]. Further the compatible system $\left\{\rho_{\wp}\right\}$ is of bounded conductor, and satisfies purity and integrality properties as in Conjecture 2 below. 
1.2. Conjectures and their discussion. Guided by the results above we make the following two conjectures. In the first we propose a reciprocity law for compatible systems of mod $\wp$ representations as the following "meta-conjecture":

Conjecture 1. Any strictly compatible L-integral system as in Definition 1 arises motivically.

To be more specific we propose the following purely Galois theoretic conjecture:

Conjecture 2. Let $\left\{\rho_{\wp}\right\}$ be a strictly compatible system as in Definition 1.

- (Lifting) It lifts to (i.e., is the reduction up to semisimplification of) a strictly compatible system of semisimple $\wp$-adic representations.

- (Bounded conductor) It is of bounded conductor, i.e., the absolute value of the norm to $\mathbf{Q}$ of the prime-to-p part of the Artin conductor of $\rho_{\wp}$ is bounded independently of $\wp$. In fact the prime-to-p part of the Artin conductor of $\rho_{\wp}$ is independent of $\wp$ for almost all $\wp$.

- (Purity) Assume that $\rho_{\wp}$ is irreducible for almost all $\wp$. Then the roots of $f_{r}(X)$ for primes $r$ not in $S$ are of absolute value $|\mathrm{Nm}(r)|^{t}$ with respect to all embeddings of $\overline{\mathbf{Q}}$ in $\mathbf{C}$, and for an integer or half-integer $t$ that is idependent of $r$. Here $\mathrm{Nm}$ is the norm map to $\mathbf{Q}$.

- (Integrality) $\left\{\rho_{\wp} \otimes \mathrm{Nm}_{\wp}^{m}\right\}$ is integral where $\mathrm{Nm}_{\wp}$ is the $\wp$-adic cyclotomic character and where $m$ is some integer.

Because of known properties of Galois representations which arise from geometry one expects that Conjecture 1 implies Conjecture 2 in the integral case. Theorem 1 proves these conjectures for 1-dimensional strictly compatible systems. Note that the first part of Conjecture 2 implies integrality of the roots of $f_{r}(X)$ at primes coprime to the residue characteristic of primes in $T$ and $r$, while the last part of the conjecture implies integrality of the roots of $f_{r}(X)$ at primes coprime to the residue characteristic of $r$. Haruzo Hida has also pointed out that one might expect that the minimal field of rationality of a strictly compatible system as in Definition 1 is totally real or CM: this is again verified in the one-dimensional case by Theorem 1 . Our conjectures may be regarded as an analog for compatible mod $p$ systems of Galois representations of the well-known conjectures of Fontaine and Mazur, cf. [FM]. Proving that a strictly compatible system $\left\{\rho_{\wp}\right\}$ arises motivically is much stronger than proving that an individual $\rho_{\wp}$ arises motivically: this is in contrast to what happens in the case of compatible systems of $\wp$-adic representations. Nevertheless in the mod $\wp$ case too, there is a link between reciprocity conjectures about the individual representations $\rho_{\wp}$ being motivic and the compatible system arising motivically, if we grant the properties of the compatible system $\left\{\rho_{\wp}\right\}$ of Conjecture 2. The reader is invited to consult [S1] where such a link is established which allows Serre to deduce the Shimura-Taniyama conjecture as a consequence of the conjectures in [S1].

Here is the plan of the paper: In Section 2 we prove a descent result (Proposition 1 below) related to the lifting property. In Section 3 we generalise a result 
of $[\mathrm{CS}]$ : this generalisation is needed for the proof of Theorem 1. The proof of Theorem 1 is carried out in Section 4.

\section{Descent results}

We prove a descent result in the context of the lifting statement of Conjecture 2. We first define what we mean by weakly compatible systems:

\section{Definition 2.}

- Let $K$ and $L$ be number fields and $S, T$ finite sets of places of $K$ and $L$ respectively. An L-rational (resp., L-integral) weakly compatible system $\left\{\rho_{\wp, \infty}\right\}$ of $n$-dimensional $\wp$-adic representations of $\mathrm{Gal}(\bar{K} / K)$ with defect $T$ and ramification set $S$, consists of giving for each finite place $\wp$ of $L$ not in $T$ a continuous semisimple representation

$$
\rho_{\wp, \infty}: G_{K} \rightarrow G L_{n}\left(L_{\wp}\right),
$$

for $L_{\wp}$ the completion of $L$ at $\wp$ whose residue field has characteristic $p$, that is

- unramified at the primes outside $S \cup\{$ places of $K$ above $p\}$

- for a place $r$ of $K$ not in $S$ there is a monic polynomial $f_{r}(X) \in$ $L[X]$ (resp., $f_{r}(X) \in \mathcal{O}_{L}[X]$ ) such that for almost all places $\wp$ of $L$, the characteristic polynomial of $\rho_{\wp, \infty}\left(\right.$ Frob $\left._{r}\right)$ is $f_{r}(X)$, where Frob $r$ is the conjugacy class of the Frobenius at $r$ in the Galois group of the extension of $K$ that is the fixed field of the kernel of $\rho_{\wp, \infty}$.

- An L-rational (resp., L-integral) weakly compatible system $\left\{\rho_{\wp}\right\}$ of $n$ dimensional mod $\wp$ representations of $\operatorname{Gal}(\bar{K} / K)$ with defect $T$ and ramification set $S$, consists of giving for each finite place $\wp$ of $L$ not in $T$ a continuous semisimple representation

$$
\rho_{\wp}: G_{K} \rightarrow G L_{n}\left(\mathbf{F}_{\wp}\right),
$$

for $\mathbf{F}_{\wp}$ the residue field of $\mathcal{O}_{L}$ at $\wp$ of characteristic $p$, that is

- unramified at the primes outside $S \cup\{$ places of $K$ above $p\}$

- for a prime $r$ of $K$ not in $S$ there is a monic polynomial $f_{r}(X) \in L[X]$ (resp., $f_{r}(X) \in \mathcal{O}_{L}[X]$ ) such that for almost all placess $\wp$ of $L$, the characteristic polynomial of $\rho_{\wp}\left(\right.$ Frob $\left._{r}\right)$ is the reduction of $f_{r}(X) \bmod$ $\wp$, where Frob $r$ is the conjugacy class of the Frobenius at $r$ in the Galois group of the extension of $K$ that is the fixed field of the kernel of $\rho_{\wp}$.

We do not know if there are weakly compatible systems in the mod $\wp$ setting that are not strictly compatible for some large but finite defect set. We prove a general proposition about lifting compatible mod $\wp$-systems $\wp$-adically.

Proposition 1. Let $K^{\prime}$ be a finite Galois extension of $K$. Consider an $L$ integral strictly compatible system $\left\{\rho_{\wp}\right\}$ of $\bmod \wp$ representations of $G_{K}:=$ $\operatorname{Gal}(\bar{K} / K)$, with the further property that $\left.\rho_{\wp}\right|_{G_{K^{\prime}}}$ is absolutely irreducible for 
almost all $\wp$. If the strictly compatible system $\left\{\left.\rho_{\wp}\right|_{G_{K^{\prime}}}\right\}$ lifts to a strictly compatible $L^{\prime}$-integral $\wp$-adic system $\left\{\rho_{\wp, K^{\prime}, \infty}\right\}$ for some number field $L^{\prime}$ with finite defect and exceptional sets, then the system $\left\{\rho_{\wp}\right\}$ lifts to a weakly compatible $L$-integral $\wp$-adic system $\left\{\rho_{\wp}, K, \infty\right\}$.

Proof. We only consider the $\rho_{\wp}$ 's for $\wp$ 's such that their residue characteristic is prime to the degree $\left[K^{\prime}: K\right]$ and for which $\left.\rho_{\wp}\right|_{G_{K^{\prime}}}$ is irreducible. Fix such a $\wp$. We assume without loss of generality that $L^{\prime}$ contains $L$, and contains all $\left[K^{\prime}: K\right]$ th roots of unity: thus we are considering $L^{\prime}$-integral compatible systems and denote the places of $L^{\prime}$ by $\wp$ etc. Then:

- $\left.\rho_{\wp}\right|_{G_{K^{\prime}}}$ lifts to $\rho_{\wp, K^{\prime}, n}$ the $\bmod \wp^{n}$ representation that is the reduction of $\rho_{\wp, K^{\prime}, \infty}$

- Note that the residue characteristic of $\wp$ is prime to the degree $\left[K^{\prime}: K\right]$, and $\left.\rho_{\wp}\right|_{G_{K^{\prime}}}$ extends to the representation $\rho_{\wp}$ of $G_{K}$. It is easy to see from this, by computing cohomological obstructions (arguing as in Proposition 1.1 of [Clo] which uses the main result of [Ca]), that for each $n$, the representation $\rho_{\wp, K^{\prime}, n}$ extends to a representation $\rho_{\wp, K, n}: G_{K} \rightarrow G L_{n}\left(\mathcal{O}_{L^{\prime}} / \wp^{n}\right)$, using that $\rho_{\wp, K^{\prime}, n}$ satisfies descent data as $\left\{\rho_{\wp, K^{\prime}, \infty}\right\}$ is a strictly compatible system, and $\left\{\rho_{\wp, K^{\prime}}\right\}$ comes by restriction from $\left\{\rho_{\wp}\right\}$.

- $\left.\rho_{\wp}\right|_{G_{K^{\prime}}}$ being irreducible, any extension of $\rho_{\wp, K^{\prime}, n}$ to $G_{K}$ is unique up to twisting by characters of $\operatorname{Gal}\left(K^{\prime} / K\right)$, and thus by twisting we can arrange that for each $n>1, \rho_{\wp, K, n}$ reduces $\bmod \wp$ to $\rho_{\wp, K, n-1}$.

Thus for all but finitely many $\wp, \rho_{\wp}$ lifts to a $\wp$-adic representation $\rho_{\wp, K, \infty}$ whose restriction to $G_{K^{\prime}}$ is $\rho_{\wp, K^{\prime}, \infty}$. Now we claim that the $\left\{\rho_{\wp, K, \infty}\right\}$ 's for such $\wp$ 's form the desired weakly compatible lift of the $\rho_{\wp}$ 's with some possibly larger defect set. First observe that $\rho_{\wp, K, \infty}$ is unramified outside primes above $p$ and a fixed finite set $S^{\prime}$ that does not depend on $p$, that we see using the fact that $K^{\prime} / K$ is ramified at only finitely many primes. Then observe that for almost all primes $r$ of $K$ there are only finitely many possibilities for the roots of the characteristic polynomial of Frob $r$ in $\rho_{\wp, K, \infty}$ as $\wp$ varies, and $r$ is fixed. This is because $\left\{\rho_{\wp}, K^{\prime}, \infty\right\}$ is in particular a weakly compatible system. Thus using the srictly compatible system $\left\{\rho_{\wp}\right\}$ it follows that the characteristic polynomial of $\rho_{\wp, K, \infty}\left(\operatorname{Frob}_{r}\right)$ is $f_{r}(X)$ for almost all primes $\wp$ and fixed $r$.

Remark. We unfortunately do not know how to prove the more desirable result that under the conditions above $\left\{\rho_{\wp}\right\}$ lifts to a strictly compatible system of $\wp-$ adic representations, although the weakly compatible $\wp$-adic system constructed above should be strictly compatible for a suitably large defect set. This is because the compatible system $\left\{\rho_{\wp}\right\}$ serves as rigidifying data in the sense that for almost all primes, more precisely all primes $\wp$ of residue characteristic prime to $\left[K^{\prime}: K\right]$, the extension $\rho_{\wp, K, \infty}$ of $\rho_{\wp, K^{\prime}, \infty}$ is determined by the requirement that it reduces to $\rho_{\wp}$ : this follows from the irreducibility hypothesis on $\left\{\left.\rho_{\wp}\right|_{G_{K^{\prime}}}\right\}$. 
We now prove a more specific descent result in the context of Conjecture 1 for 1-dimensional representations that will be useful in the proof of Theorem 1 (see Definition 4 of Section 4.1 below for unexplained terms).

Lemma 1. Let $K^{\prime}$ be a finite extension of $K$. Then an L-rational strictly compatible system $\left\{\rho_{\wp}\right\}$ of 1-dimensional mod $\wp$ representations of $G_{K}:=\operatorname{Gal}(\bar{K} / K)$ with defect $T$ and exceptional set $S$ and with bounded conductor arises from a Hecke character if and only if the strictly compatible system $\left\{\left.\rho_{\wp}\right|_{G_{K^{\prime}}}\right\}$ arises from a Hecke character.

Proof. Only one direction needs a proof. We may assume without loss of generality that $K^{\prime}$ is a Galois extension of $\mathbf{Q}$. So assume that the strictly compatible system $\left\{\rho_{\wp} \mid G_{K^{\prime}}\right\}$ arises from a Hecke character $\chi$ of the idele group of $K^{\prime}$ and

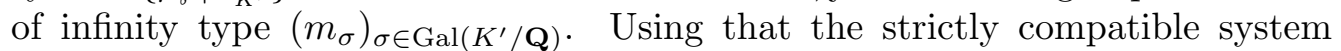
$\left\{\rho_{\wp} \mid G_{K^{\prime}}\right\}$ arises by restriction from a strictly compatible system of $G_{K}$ we see that $\chi^{\sigma}=\chi$ for all $\sigma \in \operatorname{Gal}\left(K^{\prime} / K\right)$. Consider a principal prime ideal $(a)$ of $K^{\prime}$ that lies above a prime of $\mathbf{Q}$ that splits completely in $K^{\prime}$. Then as $\chi(\sigma(a))=\chi(a)$ for all $\sigma \in \operatorname{Gal}\left(K^{\prime} / K\right)$ we deduce that $m_{\sigma}=m_{\sigma^{\prime}}$ whenever the restrictions of $\sigma, \sigma^{\prime} \in \operatorname{Gal}\left(K^{\prime} / \mathbf{Q}\right)$ to $K$ are equal, and thus for each embedding $\sigma$ of $K$ we can put without ambiguity $m_{\sigma}$ equal to $m_{\sigma^{\prime}}$ for any $\sigma^{\prime} \in \operatorname{Gal}(K / \mathbf{Q})$ that extends $\sigma$. Now consider the algebraic character $K^{*} \rightarrow \mathbf{C}^{*}$ given by $k \rightarrow \Pi_{\sigma} \sigma(k)^{m_{\sigma}}$ where $\sigma$ runs through embeddings of $K$ in $\mathbf{C}$. It is easy to see that this character is trivial on a subgroup of finite index of the units $\mathcal{O}_{K}^{*}$. Thus it is trivial by Théorème 1 of $[\mathrm{C}]$ on units congruent to $1 \bmod \mathrm{n}$ of $\mathcal{O}_{K}^{*}$ for some ideal $\mathrm{n}$ of $\mathcal{O}_{K}$. It follows that there is a Hecke character $\chi^{\prime}$ for $K$ such that $\rho_{\wp} \otimes{\tilde{\chi_{\wp}}}_{\wp}^{-1}$, where $\left\{\chi_{\wp}^{\prime}\right\}$ is the compatible mod $\wp$-system that $\chi^{\prime}$ gives rise to as described in the next section, factors through a fixed finite extension of $K$. Observe that strictly compatible systems as in Definition 1 that factor through the Galois group $\operatorname{Gal}\left(K^{\prime \prime} / K\right)$ of a fixed finite extension $K^{\prime \prime}$ of $K$ arise as reductions mod primes of a representation of $G_{K}$ into $G L_{n}(\mathcal{O})$ which factors through the Galois group of a finite extension of $K$ : here $\mathcal{O}$ the ring of integers of a number field. This finishes the proof.

We say that a compatible system $\left\{\rho_{\wp}\right\}$ as in Definition 1 arises from an Artin representation if it arises from reducing a representation $\rho: G_{K} \rightarrow G L_{n}(\mathcal{O})$ which factors through the Galois group of a finite extension of $K$, where $\mathcal{O}$ the ring of integers of a number field $L$, modulo the primes of $L$ and semisimplifying. We quote a result in Section 8 of Deligne-Serre (cf. [DS]: we thank C.S. Rajan for this reference) that characterises compatible systems $\left\{\rho_{\wp}\right\}$ that arise from Artin representations and refines the observation towards the end of the proof above.

Proposition 2 (Deligne-Serre). Let $\left\{\rho_{\wp}\right\}$ be a L-integral compatible system where we allow the defect set $T$ to be any set whose complement in the set of places of $L$ is infinite. If $\left|\operatorname{im}\left(\rho_{\wp}\right)\right|$ is bounded independently of $\wp$, then $\left\{\rho_{\wp}\right\}$ arises from an Artin representation. 


\section{Remarks.}

1. One can ask for another subtler characterisation of integral strictly compatible systems $\left\{\rho_{\wp}\right\}$ that arise from an Artin representation as those that are unramified outside a fixed finite set of places that is independent of $\wp$.

2. Compatible systems arising form Artin representations were used to prove that Serre's conjectures in [S1] imply the modularity of 2-dimensional irreducible odd complex representations of $G_{\mathbf{Q}}$ in $[\mathrm{K} 1]$.

\section{A result of Corrales and Schoof}

We need the following straightforward generalisation of Theorem 1 of [CS].

Proposition 3. Let $a_{1}, \cdots, a_{n}, c$ be a finite set of elements of $K^{*}$ and $\ell$ a rational prime. If for almost all primes $\wp$ of $K$ the subgroup generated by the image of $a_{i}$ 's in $\left(\mathcal{O}_{k} / \wp\right)^{*}$ contains $c^{t_{\wp}}$ for some integer $t_{\wp}$ that depends on $\wp$ and is prime to $\ell$, then $c^{t}=\prod_{i=1}^{i=n} a_{i}^{m_{i}}$ for some integer $t$ with $t$ prime to $\ell$, and for some integers $m_{i}, i=1, \cdots, n$. If further $t_{\wp}$ can be taken to be 1 for almost all $\wp$, then $c=\prod_{i=1}^{i=n} a_{i}^{m_{i}}$ for some integers $m_{i}, i=1, \cdots, n$.

Proof. Without loss of generality we can and will assume $\sqrt{-1} \in K$. The proof is basically the same as that of Theorem 1 of [CS]: we will briefly sketch the proof using the same breakdown into steps as in [CS].

- Step 1: Let $q$ be a power of $\ell$. Consider $K\left(\zeta_{q}, a_{1}^{1 / q}, \cdots, a_{n}^{1 / q}\right)$ and $K\left(\zeta_{q}, c^{1 / q}\right)$. Observe that for all but finitely many exceptions a prime $\wp$ of $K$ splits in $K\left(\zeta_{q}, c^{1 / q}\right)$ (resp., $K\left(\zeta_{q}, a_{1}^{1 / q}, \cdots, a_{n}^{1 / q}\right)$ ) if and only if the cardinality of the residue field at $\wp$ is $1 \bmod q$, and $c$, or equivalently $c^{t_{\wp}}$ as $t_{\wp}$ is prime to $\ell$, is a $q$ th power in $\mathbf{F}_{\wp}^{*}$ (resp., $a_{1}, \cdots, a_{n}$ are $q$ th powers in $\mathbf{F}_{\wp}^{*}$ ). Thus by assumptions of the proposition almost all primes of $K$ that split in $K\left(\zeta_{q}, a_{1}^{1 / q}, \cdots, a_{n}^{1 / q}\right)$ also split in $K\left(\zeta_{q}, c^{t_{\wp} / q}\right)=K\left(\zeta_{q}, c^{1 / q}\right)$. Thus by the Frobenius density theorem we conclude that $K\left(\zeta_{q}, c^{1 / q}\right) \subset$ $K\left(\zeta_{q}, a_{1}^{1 / q}, \cdots, a_{n}^{1 / q}\right)$.

- Step 2: Using Kummer theory and duality for finite abelian groups we deduce from Step 1 that the subgroup generated by the images of $a_{i}$ 's in $K\left(\zeta_{q}\right)^{*} / K\left(\zeta_{q}\right)^{* q}$ contains the image of $c$. From Lemma 2.1 (ii) of [CR] (see also remark on page 39 of $[\mathrm{C}]$ ), we have that the natural map $K^{*} / K^{* q} \rightarrow K\left(\zeta_{q}\right)^{*} / K\left(\zeta_{q}\right)^{* q}$ is injective, and thus we deduce that the subgroup generated by the images of $a_{i}$ 's in $K^{*} / K^{* q}$ contains the image of $c$.

- Step 3: Consider $A:=\mathcal{O}_{K, T^{\prime}}^{*} /\left\langle a_{1}, \cdots, a_{n}\right\rangle$ where $\mathcal{O}_{K, T^{\prime}}^{*}$ are the $T^{\prime}$-units with $T^{\prime}$ consisting of all places dividing any of the $a_{i}$ 's or $c$, the infinite places and the places where the hypotheses of the statement of the proposition are not satisfied. We know from Step 2 that $c$ is in $A^{q}$ for all $q$ 's that are powers of $\ell$. Using the fact that $\mathcal{O}_{K, T^{\prime}}^{*}$ is finitely generated (Dirichlet 
unit theorem), we conclude that the image of $c$ in $A$ is torsion of order prime to $\ell$ and thus we are done.

The last line of the proposition follows from the fact that assuming the $t_{\wp}$ 's to be 1 we can work with all prime powers $q$.

\section{Reciprocity for one-dimensional compatible systems}

4.1. Generalities about Hecke characters. We quickly recall the definition of Hecke characters and the association of compatible systems of $p$-adic representations to them (see Chapter II of [S], or [W] and [Tani] for details). There are equivalent ways of looking at Hecke characters that we will need below and we briefly recall these. We index as usual the real (resp., complex) places of $K$ by embeddings $\sigma$ (resp., pairs of elements $\{\sigma, c \sigma\}$ where $c$ is complex conjugation) of $K$ in C. Let $I$ be the group of ideles of $K$ and $\left(K_{\infty}^{\times}\right)^{0}$ be the connected component of the identity of the product of the completions of $K$ at the archimedean places.

Definition 3. A Hecke character $\chi$ is a continuous homomorphism $\chi: I / K^{*} \rightarrow$ $\mathrm{C}^{*}$ such that

$$
\left.\chi\right|_{\left(K_{\infty}^{\times}\right)^{0}}(x)=\Pi_{\sigma \text { real }} x_{\sigma}^{n_{\sigma}} \Pi_{\sigma \text { complex }} x_{\sigma}^{n_{\sigma}} \bar{x}_{\sigma}^{n_{c \sigma}}
$$

for integers $n_{\sigma}, n_{c \sigma}$ and with $x_{\sigma}$ the components of $x$. We say that the tuple of integers $\left(n_{\sigma}\right)_{\sigma}$ is the infinity type of $\chi$, and say that $\chi$ is unramified at a finite place $v$ if the units $U_{v}$ at $v$ are in the kernel of $\chi$. The conductor of $\chi$ is the largest ideal $\mathrm{n}$ such that elements of the finite ideles $I^{(\infty)}$ congruent to $1 \bmod \mathrm{n}$ are in the kernel of $\chi$.

From $\chi$ we get a continuous homomorphism $\chi_{0}: I /\left(K_{\infty}^{\times}\right)^{0} \rightarrow \mathbf{C}^{*}$ defined by $\chi_{0}(x)=\chi(x) \Pi_{\sigma \text { real }} x_{\sigma}^{-n_{\sigma}} \Pi_{\sigma \text { complex }} x_{\sigma}^{-n_{\sigma}} \bar{x}_{\sigma}^{-n_{c \sigma}}$ whose kernel is open and takes values in a sufficiently large subfield $L$ of $\mathbf{C}$ which is a finite extension of $\mathbf{Q}$.

For any finite place $\wp$ of $L$ above a prime $p$ of $\mathbf{Q}$ the morphism $\psi: K^{*} \rightarrow L_{\wp}^{*}$, $\psi(x)=\Pi_{\sigma} \sigma(x)^{n_{\sigma}}$, extends to a continuous morphism $\psi_{p}:\left(K \otimes \mathbf{Q}_{p}\right)^{*} \rightarrow L_{\wp}^{*}$, and from this we get a continuous character $\chi_{\wp}: I / \overline{K^{*}\left(K_{\infty}^{\times}\right)^{0}} \rightarrow L_{\wp}^{*}$ where $\chi_{\wp}=\chi_{0} \cdot\left(\psi_{p} \alpha_{p}\right)$ and $\alpha_{p}$ is the projection of $I$ to the components at places above $p$. Here $\overline{K^{*}\left(K_{\infty}^{\times}\right)^{0}}$ is the topological closure of $K^{*}\left(K_{\infty}^{\times}\right)^{0}$ in the ideles $I$. The kernel of $\chi_{\wp}$ is open in $I^{(p)}$ the ideles concentrated at places away from those dividing $p$. Using the isomorphism of class field theory $G_{K}^{a b} \simeq I / \overline{K^{*}\left(K_{\infty}^{\times}\right)^{0}}$, we see that $\left\{\chi_{\wp}\right\}$ forms a compatible system of 1 -dimensional $\wp$-adic representations of $G_{K}$ in a natural way. Since $G_{K}$ is compact, $\chi_{\wp}$ takes values in the units $\mathcal{O}_{\wp}^{*}$ of $L_{\wp}^{*}$ and thus can be reduced $\bmod \wp$.

Definition 4. We say that a strictly compatible system of (one-dimensional) mod $\wp$ representations $\left(\rho_{\wp}\right)$ as in Definition 1 arises from a Hecke character $\chi$ if $\rho_{\wp}=\tilde{\chi}_{\wp}$ where $\tilde{\chi_{\wp}}$ is the reduction of $\chi_{\wp} \bmod \wp$ at all primes $\wp$ not in $T$. 
For a fractional ideal $\mathrm{n}$ of $\mathcal{O}_{K}$, which we identify with a sequence of integers $\left(m_{v}\right)$ for $v$ running through finite places of $K$ and $m_{v}=0$ for almost all $v$, define the subgroup $U_{\mathrm{n}}$ of the ideles $I$ of $K$ to be the product $U_{v, \mathrm{n}}$ where $U_{v, \mathrm{n}}$ is the connected component of $K_{v}^{*}$ if $v$ is an infinite place, and the units $U_{v}$ congruent to 1 modulo the $m_{v}$ th power of the maximal ideal if $v$ is a finite place. Thus $K^{*} \cap U_{\mathrm{n}}$ are the totally positive units $E_{\mathrm{n}}$ of $\mathcal{O}_{K}$ congruent to $1 \bmod \mathrm{n}$. Let $I_{\mathrm{n}}$ be the quotient $I / U_{\mathrm{n}}$. We then have an exact sequence $1 \rightarrow K^{*} / E_{\mathrm{n}} \rightarrow I_{\mathrm{n}} \rightarrow C_{\mathrm{n}} \rightarrow 1$ (see loc. cit.) with $C_{\mathrm{n}}$ finite and defined by means of this exact sequence. We can consider the projective system of the $C_{\mathrm{n} p^{r}}$ 's as $r$ varies and define $C_{\mathrm{n} p^{\infty}}$ to be the projective limit. The character $\chi_{\wp}$ above maybe regarded naturally as a character of $C_{\mathrm{n} p}$ where $\mathrm{n}$ is the conductor of $\chi$.

Let $\chi$ be a Hecke character. Since the kernel of the associated homomorphism $\chi_{0}$ is open, there is a fractional ideal $\mathrm{n}$, a number field $L$ and a character $I_{\mathrm{n}} \longrightarrow$ $L^{*}$ induced by $\chi$. Viewing $K^{*}$ as an algebraic torus, the pull back to $K^{*}$ of this character is algebraic and its kernel contains $E_{\mathrm{n}}$. Conversely, a character $I_{\mathrm{n}} \longrightarrow L^{*}$ whose pull-back to $K^{*}$ is algebraic, has $E_{\mathrm{n}}$ in its kernel, and gives rise to a Hecke character. This follows from just reversing the procedure of going from $\chi$ to $\chi_{0}$ above.

It will be convenient below to switch between ideal-theoretic and ideletheoretic viewpoints. The strict ray class group $\mathrm{Cl}_{n}$ sits inside the exact sequence $1 \rightarrow P_{\mathrm{n}} \rightarrow \mathrm{Id}_{\mathrm{n}} \rightarrow \mathrm{Cl}_{\mathrm{n}} \rightarrow 1$ where $\mathrm{Id}_{\mathrm{n}}$ is the group of fractional ideals prime to $\mathrm{n}$ and $P_{\mathrm{n}}$ is the subgroup of principal fractional ideals generated by $\gamma \in K$ with $\gamma$ totally positive and congruent to $1 \bmod \mathrm{n}$. Note that we have natural maps between exact sequences

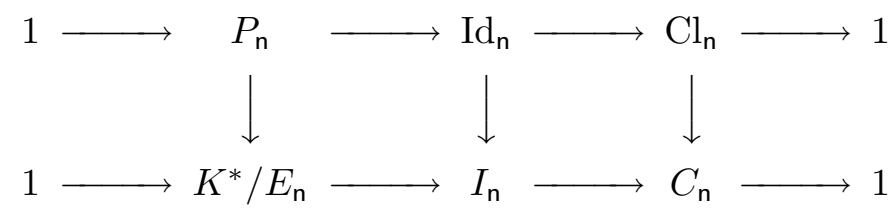

that induces an isomorphism from $\mathrm{Cl}_{\mathrm{n}}$ to $C_{\mathrm{n}}$.

4.2. Proof of Theorem 1. Let the compatible system $\left\{\rho_{\wp}\right\}$ as in Theorem 1 have defect set $T$ and ramification set $S$. The main ingredients in the proof are class field theory and Proposition 3. We first prove that the compatible system has bounded conductor, and then iterating the argument that is used to prove this, we complete the proof of the theorem.

By Lemma 1 we may assume that $K$ is a Galois extension of $\mathbf{Q}$ and has no real embeddings. Let $\mathrm{m}_{\wp}$ be the ideal of $\mathcal{O}_{K}$ that is the prime to $p$ part of the Artin conductor of $\rho_{\wp}$ for places $\wp$ of $L$ not in $T$. We assume without loss of generality that $L$ contains $K$.

A priori the fixed field of the kernel of $\rho_{\wp}$ is contained in the strict ray class field of $K$ of conductor $\mathrm{m}_{\wp} p^{\infty}$. But as the image of $\rho_{\wp}$ has order prime to $p$, the fixed field of the kernel of $\rho_{\wp}$ is in fact contained in the ray class field of conductor $\mathrm{m}_{\wp} p$. By class field theory we regard the data of the strictly compatible system as the giving of homomorphisms $\rho_{\wp}: \mathrm{Cl}_{\mathrm{m}_{\wp} p} \rightarrow \mathbf{F}_{\wp}^{*}$ where $\mathrm{Cl}_{\mathrm{m}_{\wp} p}$ is the strict ray 
class group of $K$ of conductor $\mathrm{m}_{\wp} p$, and now using the Artin map we see that the strict compatibility of the $\rho_{\wp}$ 's is expressed in terms of the images of prime ideals in $\mathrm{Cl}_{\mathrm{m}_{\wp} p}$ under $\rho_{\wp}$. We will for the most part only consider the restriction of $\rho_{\wp}: \mathrm{Cl}_{\mathrm{m}_{\wp} p} \rightarrow \mathbf{F}_{\wp}^{*}$ to the subgroup $P^{\mathrm{m}_{\wp} p} / P_{\mathrm{m}_{\wp} p}$ generated by the principal ideals $P^{\mathrm{m}_{\wp} p}$ prime to $\mathrm{m}_{\wp} p$. We can also inflate this restriction and regard it as a homomorphism of $\left(\mathcal{O}_{K} / \mathrm{m}_{\wp} p \mathcal{O}_{K}\right)^{*}$ which factors through the quotient by the image of the global units $E$.

Consider any integer $r$ of $K$ that generates a prime ideal that lies above a prime ideal of $\mathbf{Q}$ which splits completely in $K$ and is unramified in $L$. Abusing notation we will write $\rho_{\wp}(r)$ for $\rho_{\wp}((r))$ especially when we are thinking of the homomorphism of $\left(\mathcal{O}_{K} / \mathrm{m}_{\wp} p \mathcal{O}_{K}\right)^{*}$ induced by $\rho_{\wp}$. We see by the axioms of strictly compatible systems that there is an element $f_{r} \in L^{*}$ such that $\rho_{\wp}(r)$ is the reduction mod $\wp$ of $f_{r}$ that depends on $r$ but is independent of $\wp$, and is $-f_{r}(0)$ using the notation of Definition 1 . These considerations apply for all $r$ prime to $S$, and after fixing such a $r$, for all primes $\wp$ not in $S \cup T$ and such that $p$, the residue characteristic of $\wp$, does not lie below $(r)$, and is prime to $f_{r}$.

Choose a prime $\ell$ of $\mathbf{Q}$ that is coprime to the orders of the multiplicative groups of the residue fields of primes in $S$ and is prime to the residue characteristics of the prime in $S$. We claim that for almost all primes $\wp$ of $L$ (in particular we exclude the places in $T$ and the places which that lie above places ramified in $L)$ the subgroup generated by the $\{\sigma(r)\}$ 's in $\left(\mathcal{O}_{L} / \wp \mathcal{O}_{L}\right)^{*}$ contains the subgroup generated by $f_{r}^{t_{\wp}}$ (for some integer $t_{\wp}$ prime to $\ell$ ) in $\left(\mathcal{O}_{L} / \wp \mathcal{O}_{L}\right)^{*}$, and where $\sigma$ runs through the distinct embeddings of $K$ in $L$. The claim follows from considering the homomorphism of $\left(\mathcal{O}_{K} / \mathrm{m}_{\wp} p \mathcal{O}_{K}\right)^{*}\left(\simeq\left(\mathcal{O}_{K} / \mathrm{m}_{\wp} \mathcal{O}_{K}\right)^{*} \times\left(\mathcal{O}_{K} / p \mathcal{O}_{K}\right)^{*}\right)$ induced by $\rho_{\wp}$. We are using here implicitly the fact that the map $\mathcal{O}_{K} / \wp^{\prime} \rightarrow \mathcal{O}_{L} / \wp$ is injective for any prime $\wp^{\prime}$ of $K$ below $\wp$. To prove the claim note that as $\rho_{\wp}(r)$ is the reduction of $f_{r} \bmod \wp$ and $\mathrm{m}_{\wp}$ is only divisible by primes in $S$, the order of $f_{r}$ in $\mathbf{F}_{\wp}^{*}$, up to products of powers of primes which divide the orders of the multiplicative groups of the residue fields of primes in $S$ and the residue characteristics of the primes in $S$, divides the order of $r \in\left(\mathcal{O}_{K} / p \mathcal{O}_{K}\right)^{*}$. The latter, by transport of structure, is easily seen to be the l.c.m. of the orders of the $\{\sigma(r)\}_{\sigma \in \operatorname{Gal}(K / \mathbf{Q})}$ 's in $\mathbf{F}_{\wp}^{*}$.

We conclude from the claim and Proposition 3 that $f_{r}^{t(r)}=\Pi_{\sigma} \sigma(r)^{m_{r, \sigma}^{\prime}}$ for integers $t(r), m_{r, \sigma}^{\prime}$ that a priori depend on $r$, where $\sigma$ runs through the distinct embeddings of $K$ in $L$. Now as $r$ lies above a prime that is unramified in $L$, the $t(r)$ 's divide all the $m_{r, \sigma}^{\prime}$ 's. From this we conclude that we can write $f_{r}=$ $\zeta_{r} \Pi_{\sigma} \sigma(r)^{m_{r, \sigma}}$ for integers $m_{r, \sigma}$, where $\sigma$ runs through the distinct embeddings of $K$ in $L$, and for some root of unity $\zeta_{r}$. Now because of our assumption that $L$ contains $K$, and $K$ is a Galois extension of $\mathbf{Q}, \zeta_{r}$ is in $L$, and thus as $L$ contains only finitely many roots of unity, $\zeta_{r}$ has order bounded independently of $r$, and thus divides a fixed integer say $n$.

Now consider two integers $r$ and $r^{\prime}$ as above prime to $S$, that generate prime ideals that lie above distinct primes ideals of $\mathbf{Q}$ that split completely in $K$ and are unramified in $L$. Then we see as before, considering the homomorphisms of 
$\left(\mathcal{O}_{K} / \mathrm{m}_{\wp} p\right)^{*} \rightarrow \mathbf{F}_{\wp}^{*}$ induced by $\left\{\rho_{\wp}\right\}$, denoted by abuse of notation by the same symbol, that $\rho_{\wp}\left(r r^{\prime}\right)$ is the reduction $\bmod \wp$ of $f_{r} f_{r^{\prime}}$. Thus again as before it follows that $\left(f_{r} f_{r^{\prime}}\right)=\Pi_{\sigma} \sigma\left(\left(r r^{\prime}\right)\right)^{m_{r r^{\prime}, \sigma}}$ for some integers $m_{r r^{\prime}, \sigma}$. Thus we have an equality of fractional ideals of $L$ :

$$
\Pi_{\sigma} \sigma((r))^{m_{r, \sigma}} \Pi_{\sigma} \sigma\left(\left(r^{\prime}\right)\right)^{m_{r^{\prime}, \sigma}}=\Pi_{\sigma} \sigma\left(\left(r r^{\prime}\right)\right)^{m_{r r^{\prime}, \sigma}}
$$

where $\sigma$ runs through the distinct embeddings of $K$ in $L$. As $(r),\left(r^{\prime}\right)$ are split prime ideals in $K$ lying above distinct primes of $\mathbf{Q}$, we conclude that in fact the $m_{r, \sigma}=m_{r^{\prime}, \sigma}$. For conjugates $\sigma(r)$ of $r$ apply the argument above now to $\sigma(r) r^{\prime}$ and thus we conclude that the $m_{r, \sigma}$ 's are independent of $r$ and depend only on $\sigma$. We denote the common value by $m_{\sigma}$. Note that at this point we have proved $f_{r}$ is integral at all places of $L$ that have residue characteristic that is prime to the residue characteristic of $r$.

From this we easily see that the homomorphisms induced by $\rho_{\wp}^{n}$ (that we denote by the same symbol $), \rho_{\wp}^{n}:\left(\mathcal{O}_{K} / \mathrm{m}_{\wp} p \mathcal{O}_{K}\right)^{*} \rightarrow \mathbf{F}_{\wp}^{*}$ arise from the character $\chi: K^{*} \rightarrow K^{*}, \chi(x)=\Pi \sigma(x)^{n m_{\sigma}}$, by restricting $\chi$ to non-zero integral elements prime to $\mathrm{m}_{\wp} p$ and then reducing it modulo a place of $K$ below $\wp$. Thus in fact these homomorphisms $\rho_{\wp}^{n}$ factor through $\left(\mathcal{O}_{K} / p \mathcal{O}_{K}\right)^{*}$. From this we deduce that the compatible system $\left\{\rho_{\wp}\right\}$ is such that the exponents of the primes dividing the prime to $p$ part of the conductor of $\rho_{\wp}$ are bounded independently of $\wp$. As the ramification set $S$ is assumed to be finite, we conclude that the strictly compatible system $\left\{\rho_{\wp}\right\}$ has bounded conductor in the sense of Definition 1.

We deduce that the ideals $\mathrm{m}_{\wp}$ all contain a fixed non-zero ideal $\mathrm{m}$, and thus we can regard the homomorphisms $\rho_{\wp}$ as homomorphisms from $\mathrm{Cl}_{\mathrm{m} p}$. At this point, essentially we have to repeat the argument above. Consider principal prime ideals $(r)$ with $r$ congruent to $1 \mathrm{mod} \mathrm{m}$ that lie above primes that split completely in $K$. Repeat the argument above to get that this time $f_{r}$, which we know a priori is in $K$ using the Frobenius density theorem, is $=\Pi_{\sigma} \sigma(r)^{m_{\sigma}}$ for the same $m_{\sigma}$ 's as above, using the last sentence of Proposition 3. Now observe that the algebraic character $K^{*} \rightarrow K^{*}$ that sends $x$ to $\Pi_{\sigma} \sigma(x)^{m_{\sigma}}$ is trivial on units congruent to $1 \bmod \mathrm{m}$. Then using the facts recalled in Section 4.1, and that such $r$ 's, prime to $p$, project surjectively to $\left(\mathcal{O}_{K} / p \mathcal{O}_{K}\right)^{*}$ for $p$ prime to $\mathrm{m}$, we deduce by the axioms of strictly compatible systems, that there is a Hecke character $\chi$ of infinity type $\left(m_{\sigma}\right)$, such that $\rho_{\wp} \otimes \tilde{\chi}_{\wp}^{-1}$ factors through the Galois group of a fixed finite extension of $K$ : we may take this finite extension to be the strict ray class field of conductor $m$. The proof of the theorem is now complete by appealing to Lemma 1.

\subsection{Some remarks.}

1. The proof of Theorem 1 follows the general lines of the method of $[\mathrm{K}]$ that dealt with the case $K=\mathbf{Q}$. The presentation of the proof in $[\mathrm{K}]$ is inaccurate when $S$ is non-empty, as the second line of the proof is unjustified (we thank N. Fakhruddin for pointing this out): nevertheless the proof of loc. cit. can be modified without much difficulty to work in the general 
case considered there of compatible systems of bounded conductor. As compared to $[\mathrm{K}]$, the substantive improvements made in this paper as far as the results about one-dimensional systems are concerned, are that we no longer assume $K=\mathbf{Q}$, we no longer have a bounded conductor hypothesis, and the the proof is simplified to the extent that we no longer appeal to results towards Artin's conjecture on primitive roots.

2. In [S2] a similar theorem was proved assuming that "inertial weights" (see Section 1.7 of [S3]) of the $\rho_{\wp}$ 's were bounded independently of $\wp$. In our proof the fact that inertial weights are bounded is proved to be a consequence of the defining properties of a compatible system.

3. The proof works even if we allow the set $T$ in Definition 1 to be a set of places of density 0.

4. The proof also gives that one-dimensional weakly compatible mod $\wp$ systems are (weakly) equivalent in a natural sense to strictly compatible systems that arise from a uniquely determined Hecke character.

5. Although our conjectures seem inaccessible at the moment in the higher dimensional situation, it will be of interest to prove some more accessible "independence of $p$ " results for the images of $\rho_{\wp}\left(G_{K}\right)$ analogous to the case of compatible $\wp$-adic systems studied in [LP]. For instance one might expect that for 2-dimensional strictly compatible systems either for a set of primes $\wp$ of density $1, \operatorname{im}\left(\rho_{\wp}\right)$ has an abelian subgroup of bounded index, or for a set of primes $\wp$ of density $1, \operatorname{im}\left(\rho_{\wp}\right)$ contains $S L_{2}\left(\mathbf{F}_{p}\right)$. This will be a necessary step in studying the adelic images of compatible systems of $p$-adic representations in the abstract case studied in [LP].

6. The first step in tackling the conjectures of the introduction for dimensions greater than 1, would be to generalise Theorem 1 and prove that abelian semisimple compatible mod $\wp$ systems of $G_{K}$ are sums of 1-dimensional compatible mod $\wp$ systems that arise from Hecke characters. One can indeed prove this by suitably modifying the methods of this paper.

\section{Acknowledgements}

I thank Najmuddin Fakhruddin for his interest, and for the many interesting conversations we've had about compatible systems. I thank the referee for useful suggestions to improve the paper.

\section{References}

[C] C. Chevalley, Deux théorèmes d'arithmétique, J. Math. Soc. Japan 3 (1951), 36-44.

[Ca] H. Carayol, Formes modulaires et représentations galoisiennes avec valeurs dans un anneau local complet, $p$-adic monodromy and the Birch and Swinnerton-Dyer conjecture (Boston, MA, 1991), 213-237, Contemp. Math. 165, Amer. Math. Soc., Providence, RI, 1994.

[Clo] L. Clozel, Sur la théorie de Wiles et le changement de base nonabélien, Internat. Math. Res. Notices 1995, 437-444. 
[CS] C. Corrales-Rodrigáñez, R. Schoof, The support problem and its elliptic analogue, J. Number Theory 64 (1997), 276-290.

[DS] P. Deligne, J.-P. Serre, Formes modulaires de poids 1, Ann. Sci. École Norm. Sup. (4) 7 (1974), 507-530.

[FM] J.-M. Fontaine, B. Mazur, Geometric Galois representations, Elliptic curves, modular forms, \& Fermat's last theorem (Hong Kong, 1993), 41-78, Ser. Number Theory, I, International Press, Cambridge, MA, 1995.

[H] G. Henniart, Représentations $\ell$-adiques abéliennes, Seminar on Number Theory, Paris 1980-81 (Paris, 1980/1981), 107-126, Progr. Math., 22, Birkhäuser Boston, Boston, MA, 1982.

[K] C. Khare, Compatible systems of $\bmod p$ Galois representations, C. R. Acad. Sci. Paris Sér I Math. 323 (1996), 117-120.

[K1] , Remarks on mod p forms of weight one, Internat. Math. Res. Notices 1997, 127-133. (Corrigendum: Internat. Math. Res. Notices 1999, 1029-1030.)

[LP] M. Larsen, R. Pink, On $\ell$-independence of algebraic monodromy groups in compatible systems of representations, Invent. Math. 107 (1992), 603-636.

[S] J.-P. Serre, Abelian $\ell$-adic representations and elliptic curves, Second edition. Advanced Book Classics. Addison-Wesley Publishing Company, Advanced Book Program, Redwood City, CA, 1989.

[S1] $\quad$ Sur les représentations modulaires de degré 2 de $\operatorname{Gal}(\overline{\mathbf{Q}} / \mathbf{Q})$, Duke Math. J. 54 (1987), 179-230.

[S2] _ Résumés des cours de 1970-71, Oeuvres, Vol. II, no. 93.

[S3] Propriétés galoisiennes des points d'ordre finides courbes elliptiques, Invent. Math. 15 (1972), 259-331.

[Sch] N. Schappacher, Periods of Hecke characters, Lecture Notes in Mathematics, 1301. Springer-Verlag, Berlin, 1988.

[Tani] Y. Taniyama, L-functions of number fields and zeta functions of abelian varieties, J. Math. Soc. Japan 9 (1957), 330-366.

[W] A. Weil, On a certain type of characters of the idele-class group of an algebraic number field, Proceedings of the international symposium on algebraic number theory, Tokyo \& Nikko, 1955, pp. 1-7. Science Council of Japan, Tokyo, 1956.

School of Mathematics, TifR, Homi Bhabha Road, Mumbai 400 005, India.

E-mail address: shekhar@math.tifr.res.in

Dept of Math, University of Utah, 155 S 1400 E, Salt Lake City, UT 84112, U.S.A.

E-mail address: shekhar@math.utah.edu 